OPEN ACCESS

Edited by: Ying $X u$,

The State University of New York at Buffalo, USA

Reviewed by: Guido Gainotti, Policlinico Gemelli, Italy Ramesh Kandimalla, Emory University, USA

*Correspondence: Agnes S. Chan aschan@psy.cuhk.edu.hk

Received: 03 February 2016 Accepted: 09 March 2016 Published: 29 March 2016

Citation:

Yeung MK, Sze SL, Woo J, Kwok T, Shum DHK, Yu R and Chan AS (2016) Altered Frontal Lateralization Underlies the Category Fluency Deficits in Older Adults with Mild Cognitive Impairment: A Near-Infrared Spectroscopy Study.

Front. Aging Neurosci. 8:59.

doi: 10.3389/fnagi.2016.00059

\section{Altered Frontal Lateralization Underlies the Category Fluency Deficits in Older Adults with Mild Cognitive Impairment: A Near-Infrared Spectroscopy Study}

\author{
Michael K. Yeung ${ }^{1}$, Sophia L. Sze ${ }^{1,2}$, Jean Woo ${ }^{3}$, Timothy Kwok ${ }^{4}$, David H. K. Shum ${ }^{5}$, \\ Ruby $\mathrm{Yu}^{3}$ and Agnes S. Chan ${ }^{1,2 *}$
}

\begin{abstract}
Department of Psychology, The Chinese University of Hong Kong, New Territories, Hong Kong, China, ${ }^{2}$ Chanwuyi Research Center for Neuropsychological Well-Being, The Chinese University of Hong Kong, New Territories, Hong Kong, China, ${ }^{3}$ Department of Medicine and Therapeutics, The Chinese University of Hong Kong, New Territories, Hong Kong, China, ${ }^{4}$ School of Public Health, The Chinese University of Hong Kong, New Territories, Hong Kong, China, ${ }^{5}$ Menzies Health Institute Queensland and School of Applied Psychology, Griffith University, Gold Coast, QLD, Australia
\end{abstract}

Individuals with mild cognitive impairment ( $\mathrm{MCl}$ ) have been consistently found to have category fluency deficits. However, little is known about the neural basis of these deficits. A diversity of neuroimaging studies has revealed left-lateralized prefrontal activations due to verbal processing and control functions during the performance of category fluency tasks. Given the reports of structural and functional abnormalities in the prefrontal cortices in individuals with $\mathrm{MCl}$, it is conceivable that these individuals would also exhibit altered prefrontal activation patterns during a category fluency task. The present study aimed to investigate the prefrontal dynamics during the category fluency task in older adults with $\mathrm{MCl}$ by using near-infrared spectroscopy (NIRS). Twenty-six older adults with $\mathrm{MCl}$ were compared with 26 older adults with normal cognition (NC) who were matched in age, gender, handedness, and educational level. All participants performed a category fluency task while the prefrontal dynamics were recorded. The results showed that the $\mathrm{MCl}$ group generated fewer unique words, made fewer switches between subcategories, and generated fewer new subcategories than did the NC group. Importantly, the NIRS results showed that the NC group exhibited a left lateralization of frontal activations during the category fluency task, while the $\mathrm{MCl}$ group did not exhibit such a lateralization. Furthermore, there was a significant positive correlation between the category fluency performance and the extent of lateralization, suggesting that the category fluency deficits in the $\mathrm{MCl}$ group could be related to frontal dysfunction. That is, the rightward shift of frontal activations in the $\mathrm{MCl}$ group may reflect the presence of cortical reorganization in which the contralateral regions (i.e., the right hemisphere) are recruited to take over the function that is declining in the specialized regions (i.e., the left hemisphere). Our lateralization finding may serve as an objective neural marker for distinguishing between normal aging and $\mathrm{MCl}$. Our study highlights that an alteration of neural functioning is already present at the prodromal stage of dementia.

Keywords: mild cognitive impairment $(\mathrm{MCl})$, verbal fluency, category fluency, prefrontal cortex, near-infrared spectroscopy (NIRS), lateralization 


\section{INTRODUCTION}

Mild cognitive impairment (MCI) is considered to be a transitional stage between healthy aging and dementia. Individuals with MCI are concerned about a cognitive decline and exhibit objective cognitive impairment but have essentially normal activities of daily living and are not demented (Albert et al., 2011; Petersen et al., 2014). The MCI construct is heterogeneous. Based on the presence of memory deficits, MCI can be broadly classified into the amnestic and nonamnestic subtypes (Petersen et al., 2014), which have been found to be associated with different etiologies (Yaffe et al., 2006). With regard to neuroanatomy, the amnestic subtype has major disturbances in medial temporal lobe structures, while non-amnestic subtype has major disturbances in other brain structures, such as the frontal cortex when executive dysfunction is evident (e.g., Chao et al., 2009; Pa et al., 2009). The conversion rate of MCI to dementia has been estimated to be approximately $10-20 \%$ per annum (Petersen et al., 1999; Amieva et al., 2004; Venneri et al., 2011; Wang et al., 2011). Because early detection of individuals at the prodromal stage of dementia offers the best potential for intervention (Ernst et al., 1997), it is important to identify objective markers, such as neuropsychological and neurophysiological characteristics that can reliably distinguish MCI from normal aging.

The category fluency test is a commonly used neuropsychological test that requires individuals to generate as many words as possible that belong to a semantic category (e.g., animal names) within a given time interval. This task requires the initiation of a specific verbal behavior, a controlled and strategic search of specific verbal information, and selfmonitoring to avoid intrusions and repetitions (Bertola et al., 2014). The test has been found to be a significant predictor of future cognitive decline in both the cognitively normal (e.g., Tierney et al., 2005) and MCI populations (e.g., Molinuevo et al., 2011; Venneri et al., 2011). Numerous empirical studies have consistently found that individuals with MCI, irrespective of their subtypes, exhibited category fluency impairment (Murphy et al., 2006; Nutter-Upham et al., 2008; Brandt and Manning, 2009; Libon et al., 2010; Wang et al., 2011; Price et al., 2012; Weakley et al., 2013; Bertola et al., 2014; Rinehardt et al., 2014; Vogel et al., 2014). This impairment has been mainly attributed to their deficits in generating new subcategories or switching between subcategories (Raoux et al., 2008; Price et al., 2012; Weakley et al., 2013; Bertola et al., 2014), although impairment in clustering (Price et al., 2012) may also underlie their fluency deficits. In sum, these findings provide strong evidence for the presence of a category fluency deficit in individuals with MCI.

Category fluency has been investigated extensively using neuroimaging techniques, such as positron emission topography (PET; e.g., Gourovitch et al., 2000), functional magnetic resonance imaging (fMRI: e.g., Weiss et al., 2003; Birn et al., 2010; Meinzer et al., 2013; for a recent meta-analysis, see Wagner et al., 2014), near-infrared spectroscopy (NIRS; Schecklmann et al., 2007; Tupak et al., 2012; Dan et al.,
2013; Heinzel et al., 2013, 2015; Liu et al., 2014; Marumo et al., 2014; Holper et al., 2015), and functional transcranial Doppler sonography (fTCD; Gutierrez-Sigut et al., 2015). These studies have consistently found that the prefrontal cortices activate during the performance of category fluency tasks in healthy individuals, reflecting the control functions exerted by the (left inferior) prefrontal cortex in semantic tasks (Thompson-Schill et al., 1997). In addition, a majority has reported a left lateralization of these frontal activations (e.g., Gourovitch et al., 2000; Weiss et al., 2003; Birn et al., 2010; Tupak et al., 2012; Gutierrez-Sigut et al., 2015; Heinzel et al., 2015), most likely reflecting the general dominance of the left hemisphere in verbal functioning (Hellige, 1993). In addition, the absence of a left frontal lateralization (i.e., bilateral or right-dominant activation patterns) during verbal tasks has been found in some disorders and diseases that exhibit verbal or category fluency impairment, including Alzheimer's dementia (Fallgatter et al., 1997), schizophrenia (Sommer et al., 2001; Weiss et al., 2004), and developmental dysphasia (de Guibert et al., 2011). A focal left-brain injury has also been shown to result in category fluency deficits in addition to a selective reduction of left frontal lateralization during performance (Raja Beharelle et al., 2010). In sum, left-lateralized frontal activations have been observed during category fluency performance, and a reduction or a reversal of lateralization has been documented in individuals with neurocognitive impairment.

Recent neuroimaging studies have revealed frontal abnormality in individuals with MCI. Specifically, some MRI studies have reported a cortical atrophy in the (left) prefrontal cortices in individuals with amnestic or non-amnestic MCI (Pa et al., 2009; Wang et al., 2009; Chang et al., 2010; Ahn et al., 2011; Zhao et al., 2015). In addition, a link between poor semantic fluency performance and cortical atrophy of the lateral prefrontal regions (especially in the left hemisphere) has also been reported in these individuals (Ahn et al., 2011; Eastman et al., 2013; Clark et al., 2014). Furthermore, some fMRI studies have shown that these individuals exhibit abnormal frontal activations while performing various cognitive tasks, including episodic memory retrieval (Heun et al., 2007), semantic memory (Woodard et al., 2009), divided attention (Dannhauser et al., 2005), interference control (Kaufmann et al., 2008; Van Dam et al., 2013), and working memory (Alichniewicz et al., 2012; Papma et al., 2013; see Li et al., 2015, for a recent meta-analysis of fMRI studies in MCI). In summary, structural and functional abnormalities in the prefrontal cortices have been reported in the MCI population, regardless of the subtype.

Given that category fluency seems to be mediated by the frontal lobe and that individuals with MCI have shown frontal dysfunction, it is reasonable to hypothesize that individuals with MCI will demonstrate abnormal frontal activations during category fluency performance. In addition, a reduction in leftlateralized frontal activations has been shown as an indicator of frontal dysfunction during the fluency task. Thus, it is anticipated that individuals with MCI will demonstrate a reduced lateralization of frontal activations during the category fluency 
task. As a further investigation, we would compare the amnestic and non-amnestic MCI subtypes given that the MCI construct is heterogeneous (Petersen et al., 2014).

The NIRS is an optical imaging method that uses light in the near-infrared spectrum (700-1000 nm) to noninvasively monitor the hemodynamic responses evoked by brain activity (Villringer and Chance, 1997). It measures the quantitative changes of the oxygenated hemoglobin (oxy-Hb) and deoxygenated hemoglobin (deoxy-Hb) concentration in the cerebral blood. These NIRS signals have been shown to correlate with the blood oxygenation level dependent (BOLD) signals as measured by the fMRI (Strangman et al., 2002; Cui et al., 2011; Sato et al., 2013). The NIRS has been extensively used to study the prefrontal brain dynamics during the category fluency task in healthy adults (Hori et al., 2008; Kahlaoui et al., 2012; Tupak et al., 2012; Dan et al., 2013; Heinzel et al., 2013, 2015) and those with psychiatric disorders or neurological diseases, including schizophrenia (Kubota et al., 2005; Ehlis et al., 2007; Marumo et al., 2014), major depression disorder (Liu et al., 2014), and Alzheimer's Disease (Fallgatter et al., 1997). Previous NIRS studies with healthy adults have consistently found [oxy-Hb] increases in the prefrontal cortices (predominantly lateral regions) during the category fluency task (Schecklmann et al., 2007; Tupak et al., 2012; Dan et al., 2013; Heinzel et al., 2013, 2015; Liu et al., 2014; Marumo et al., 2014; Holper et al., 2015). In addition, many of these have reported a left lateralization of [oxy-Hb] increases at the lateral prefrontal regions (Fallgatter et al., 1997; Tupak et al., 2012; Dan et al., 2013; Heinzel et al., 2013, 2015) and thus are largely consistent with the fMRI literature (fMRI: e.g., Weiss et al., 2003; Meinzer et al., 2013; for review, see Wagner et al., 2014; fTCD: Gutierrez-Sigut et al., 2015). Therefore, accumulating evidence has supported the use of NIRS in studying the prefrontal dynamics during the category fluency task, in which left-lateralized frontal activations (i.e., [oxy-Hb] increases) could be detected (e.g., Tupak et al., 2012; Dan et al., 2013; Heinzel et al., 2015). Thus, the present study utilized NIRS to study the frontal activations associated with category fluency in individuals with MCI.

\section{MATERIALS AND METHODS}

\section{Participants}

The original sample included 69 older adults who were aged between 60 and 91 years. The participants were recruited through advertisements in the community and from the health and social centers in the New Territories East regions in Hong Kong. They were invited to engage in an intervention program on memory. As a pre-intervention assessment, all of the participants underwent a comprehensive neuropsychological assessment and a NIRS recording session. Exclusion criteria were as follows: (1) a score higher than 7 on the short form of the Chinese Geriatric Depression Scale (CGDS-SF; Lee et al., 1993); (2) a score higher than 15 (i.e., in the moderate or severe range) on the Beck Anxiety Inventory (BAI; Beck et al., 1988); (3) a history of head injury or neurological/psychiatric disorders; and (4) signs of dementia (e.g., impaired on all neuropsychological measures). Following the criteria proposed by Petersen et al. (2014) and the National Institute on Aging-Alzheimer's Association workgroups (Albert et al., 2011), participants were considered to have MCI if they met the following criteria: (1) a concern regarding a change in cognition; (2) an objective cognitive impairment; (3) a preservation of independence in functional abilities; and (4) not being demented. The objective cognitive impairment was operationalized in accordance with the comprehensive neuropsychological criteria proposed by Jak et al. (2009), in which participants were considered to have MCI if they scored more than 1 SD below the age- (and education-) corrected normative mean on at least two measures within at least one cognitive domain (i.e., memory, speed/executive function, language, and visuospatial function; see Table 1). The use of these criteria, instead of a single impaired score, has been shown to reduce false positive errors in detecting MCI (Clark et al., 2013; Bondi et al., 2014). On the other hand, participants were considered to be cognitively normal if, at most, performance on one measure within one or two cognitive domains fell more than $1 \mathrm{SD}$ below the age- (and education-) corrected norms (Jak et al., 2009).

The final sample consisted of 26 older adults with MCI and 26 older adults with normal cognition (NC) after excluding participants who were unable to complete the tests in the NIRS recording session and matching the two groups on mean age and educational level, gender, and handedness $(p>0.10)$. According to Petersen et al. (2014), 10 older adults with MCI belonged to the amnestic subtype, and 16 belonged to the non-amnestic subtype. Because there were no significant differences between the two MCI groups except for memory measures, $p s>0.05$, and because the sample size of each group was small, we mainly present the results with the combined MCI group. The demographic and neuropsychological characteristics of the NC and MCI groups are shown in Table 2. All of the participants provided written informed consent prior to the study. The study was approved by the Joint Chinese University of Hong Kong-New Territories East Cluster (CUHK-NTEC) Clinical Research Ethics Review Committee.

\section{Measures and Procedure}

For the neuropsychological assessment, all of the participants underwent a neuropsychological protocol that included the following tests: the Hong Kong List Learning Test (HKLLT; Chan and Kwok, 2006) and the Visual Reproduction of the Wechsler Memory Scale (WMS-VR; Wechsler, 2005) as the memory tests; The Shape Trail Test (STT; Zhao et al., 2013) and the Rey-Osterrieth Complex Figure Test (RCFT; Bernstein and Waber, 1996) as the tests for speed/executive function; the Boston Naming Test (BNT; Cheung et al., 2004) as the test for language; and the WMS-VR and RCFT as the tests for visuospatial function. In addition, the Chinese version of the Mattis Dementia Rating Scale (CDRS; Chan et al., 2001) was also administered to estimate the global functioning level. The short form of the Chinese Geriatric Depression Scale (CGDS-SF) and the Beck Anxiety Inventory (BAI) were used to assess depression 
TABLE 1 | Neuropsychological measures used in the present study.

\begin{tabular}{|c|c|c|c|}
\hline Memory & Speed/executive function & Language & Visuospatial function \\
\hline HKLLT: total learning & STT-A: time & CF (animals): total unique words in $60 \mathrm{~s}$ & WMS-VR: copy \\
\hline HKLLT: delayed recall & STT-B: time & CF (transportation): total unique words in $60 \mathrm{~s}$ & RCFT: copy score \\
\hline $\begin{array}{l}\text { WMS-VR: immediate recall } \\
\text { WMS-VR: delayed recall }\end{array}$ & RCFT: copy organization & BNT: spontaneous naming & \\
\hline
\end{tabular}

Note: BNT, Boston Naming Test; CF, Category Fluency; HKLLT, Hong Kong List Learning Test; RCFT, Rey-Osterrieth Complex Figure Test; STT, Shape Trail Test; WMS-VR, Wechsler Memory Scale-Visual Reproduction. An objective cognitive impairment for mild cognitive impairment (MCl) was operationalized by the scoring of more than 1 SD below the age- (and education-) corrected normative mean on at least two measures within at least one cognitive domain (i.e., memory, speed/executive function, language, and visuospatial function).

TABLE 2 | Demographic and neuropsychological characteristics of the mild cognitive impairment (MCI) and normal cognition (NC) groups.

\begin{tabular}{|c|c|c|c|c|}
\hline & \multicolumn{2}{|c|}{ Group } & \multirow[b]{2}{*}{$t / \Lambda$} & \multirow[b]{2}{*}{$p$} \\
\hline & $\mathrm{MCl}(n=26)$ & $\mathrm{NC}(n=26)$ & & \\
\hline Age (yrs) & $69.07(6.20)$ & $68.87(6.08)$ & 0.12 & 0.91 \\
\hline Gender (M/F) & $6 / 20$ & $7 / 19$ & 0.10 & 0.75 \\
\hline Handedness (R/L) & $25 / 1$ & $25 / 1$ & 0.00 & 1.00 \\
\hline Education (yrs) & $7.94(4.41)$ & $9.58(3.26)$ & 1.52 & 0.14 \\
\hline \multicolumn{5}{|l|}{ Memory } \\
\hline HKLLT: total learning & $20.92(6.42)$ & $24.00(5.50)$ & 1.86 & $0.069^{\dagger}$ \\
\hline HKLLT: delayed recall & $6.35(2.87)$ & $8.08(2.95)$ & 2.14 & $0.037^{*}$ \\
\hline WMS-VR: immediate recall & $61.04(15.96)$ & $71.62(10.16)$ & 2.85 & $0.006^{* *}$ \\
\hline WMS-VR: delayed recall & $36.62(17.41)$ & $51.15(21.89)$ & 2.65 & $0.011^{*}$ \\
\hline \multicolumn{5}{|l|}{ Speed/executive functions } \\
\hline STT-A: time (s) & 69.19 (21.99) & $54.54(16.03)$ & 2.75 & $0.008^{* *}$ \\
\hline STT-B: time (s) & $216.27(108.75)$ & $140.00(49.80)$ & 3.25 & $0.003^{* *}$ \\
\hline RCFT: copy organization & $5.50(2.29)$ & $6.85(1.26)$ & 2.63 & $0.012^{*}$ \\
\hline \multicolumn{5}{|l|}{ Language } \\
\hline CF (animals): unique words & $14.04(4.64)$ & $18.00(5.03)$ & 2.95 & $0.005^{* *}$ \\
\hline CF (transportation): unique words & $10.15(2.96)$ & $11.92(2.83)$ & 2.2 & $0.032^{*}$ \\
\hline BNT: spontaneous naming & $17.15(4.05)$ & $21.04(2.27)$ & 4.27 & $<0.001^{* * *}$ \\
\hline \multicolumn{5}{|l|}{ Visuospatial functions } \\
\hline WMS-VR: copy & $91.35(5.38)$ & $95.38(4.36)$ & 2.97 & $0.005^{* *}$ \\
\hline RCFT: copy score & $28.54(7.83)$ & $32.75(2.46)$ & 2.62 & $0.014^{*}$ \\
\hline \multicolumn{5}{|l|}{ Global functioning level } \\
\hline CDRS: adjusted total score & $149.34(6.67)$ & $153.44(6.25)$ & 2.27 & $0.028^{*}$ \\
\hline \multicolumn{5}{|l|}{ Questionnaires } \\
\hline ADL-PI-Self: daily functioning & 38.67 (4.94) & $38.00(4.13)$ & 0.51 & 0.61 \\
\hline ADL-PI-Self: physical functioning & $4.88(0.34)$ & $4.88(0.34)$ & 0.00 & 1.00 \\
\hline $\mathrm{BAl}$ & $2.73(2.99)$ & $4.15(3.60)$ & 1.55 & 0.13 \\
\hline CGDS-SF & $2.12(1.93)$ & $2.62(2.08)$ & 0.90 & 0.37 \\
\hline
\end{tabular}

Note: ADL-PI-Self, Self-report version of the Activities of Daily Living-Prevention Instrument; BAl, Beck Anxiety Inventory; BNT, Boston Naming Test; CDRS, Chinese Version of the Mattis Dementia Rating Scale; CF, Category Fluency; CGDS, Chinese Geriatric Depression Scale-Short Form; HKLLT, Hong Kong List Learning Test; RCFT, Rey-Osterrieth Complex Figure Test; STT, Shape Trail Test; WMS-VR, Wechsler Memory Scale-Visual Reproduction. There were four missing values for the ADL-PI-Self (MCl: $n=2 ; H C: n=2)$, and 1 for the CDRS (NC: $n=1)$. Standard deviations are in parentheses. ${ }^{\dagger} p<0.10 ; *^{*} p<0.05 ;{ }^{* *} p<0.01 ;{ }^{* * *} p<0.001$.

and anxiety levels. The self-report version of the Activities of Daily Living-Instrument Prevention (ADL-PI-Self; Galasko et al., 2006) was used to assess daily and physical functioning levels. The assessment was administered by trained research assistants.

The design of the category fluency task was adapted from previous NIRS studies of verbal fluency (e.g., Tupak et al., 2012; Liu et al., 2014) in which the task blocks were interleaved with control blocks (i.e., a blocked design). For the task blocks, the participants had to generate words that belong to a particular semantic category, of which the category word was shown at the center of a computer screen. Animal and means of transportation were employed as the categories for word generation in a fixed order in accordance with a previous Chinese normative study (Chan and Poon, 1999) that would allow the conversion of $Z$ scores for MCI classification. For the control blocks, the participants had to slowly repeat " $1,2,3,4$ ", of which the phrase was shown at the center of the screen, to control for the effect of overt verbal production. 
Before the experiment began, participants were first briefed about the task instructions and experimental design, and flower words were used as the category example. They were asked to generate as many words as possible during the task blocks. They were also asked to open their eyes and to avoid making any movements throughout the experiment. The experiment began with a 30-s control period, followed immediately by a 60-s task period (i.e., animal words), a 60-s control period, another 60 -s task period (i.e., transportation words), and ended with another 60-s control period. The total measurement period was 270 s. The stimuli were presented using the E-Prime 1.2 Software (Psychology Software Tools, Pittsburgh, PA, USA). The NIRS session was administered by a doctorate student and a trained research assistant.

\section{NIRS Measurement}

The relative [oxy-Hb] and [deoxy-Hb] changes were recorded using a 16-channel OEG-SpO2 system (Spectratech Inc., Tokyo, Japan). The machine uses two wavelengths of near-infrared light, 770 and $840 \mathrm{~nm}$, and calculates the amount of absorbed near-infrared light based on the modified Beer-Lambert Law (Delpy et al., 1988). Six emission and six detector probes were arranged in a 2 rows $\times 6$ columns matrix on the participant's forehead (see Figure 1). The distance between pairs of emitter and detector probes was $3 \mathrm{~cm}$, thus measuring the [oxy- $\mathrm{Hb}$ ] changes at a depth of $2-3 \mathrm{~cm}$ below the scalp (Toronov et al., 2001). The NIRS data were collected at 16 measurement points that were located in between each pair of emitter and detector probes. In accordance to the international 10/20 system (Jasper, 1958), the center of the probe matrix was placed on Fpz, and the probes at the bottom left and right corners were placed around F7 and F8, respectively. The sampling rate was $12.21 \mathrm{~Hz}$. Because the [oxy-Hb] signals have been shown to have a better signal-to-noise ratio and demonstrate a stronger relationship with the BOLD signal as measured by the fMRI or with the cerebral blood flow compared to the [deoxy-Hb] signals (Strangman et al., 2002; Cui et al., 2011), we investigated only the [oxy-Hb] data in the present study.



FIGURE 1 | Arrangement of the near-infrared spectroscopy (NIRS) channels. Channels 13-16 represent the left prefrontal region, and channels 1-4 represent the right prefrontal region. The distance between pairs of emitter and detector probes was $3 \mathrm{~cm}$. In accordance to the international 10/20 system, the center of the probe matrix was placed on Fpz, and the probes at the bottom left and right corners were placed around F7 and F8, respectively.

\section{Data Analysis}

For the non-categorical demographic and neuropsychological data, the MCI and NC groups were compared using independent sample $t$-tests. For the categorical variables (i.e., gender and handedness), the groups were compared using the Likelihood Ratio tests. We also further investigated the cognitive processes (i.e., clustering and switching) underlying the animal fluency task as proposed by Troyer (2000). Clustering is an automatic process that involves the generation of words within semantic subcategories and reflects semantic associations, whereas switching is an effortful executive process that reflects flexible thinking. The scoring procedure and listing can be found in Troyer. In brief, the mean cluster size referred to the mean number of successively generated words that belong to a same subcategory, such as African animals, canines, etc., including intruded and repeated words. Clustering starts with a second successive word. For example, two related words constitute a cluster size of 1 , or three related words constitute a cluster size of 2 . The number of switches refers to the number of switches between subcategories. New subcategory generation, which measures the ability to access semantic subcategories, was also investigated (Price et al., 2012; Bertola et al., 2014).

For the [oxy-Hb] data, a low-pass filter at $0.10 \mathrm{~Hz}$ and a slope of $60 \mathrm{~dB} /$ octave was first applied to remove short-term motion and cardiac artifacts. Then, we performed a first-order linear fitting, based on the mean values of the pre- and posttask baseline periods, defined by the $10 \mathrm{~s}$ preceding the 60 -s task block and the last $10 \mathrm{~s}$ of the following control block, respectively, to exclude the task-unrelated changes during the two 60-s task blocks. The baseline-corrected data were then averaged across time points for each fluency condition, channel, and participant. The baseline correction and averaging were performed using Matlab ${ }^{\circledR}$ R2014a (The MathWorks, Natick, MA, USA). Because both fMRI (Wagner et al., 2014) and NIRS (e.g., Tupak et al., 2012) studies have shown the lateral prefrontal cortex as the main site of activation during the category fluency task, we conducted a region-of-interest (ROI) analysis: channels 13-16 represent the left prefrontal region, and channels 1-4 represent the right (Makizako et al., 2013).

To quantify the lateralization of frontal activations (i.e., [oxy-Hb] increases), we adopted a modified version of the laterality index that is formulated as $\left(A_{L}-A_{R}\right) /\left(\left|A_{L}\right|+\left|A_{R}\right|\right.$; Seghier, 2008). $A_{L}$ is the mean [oxy-Hb] changes on the left side, and $A_{R}$ is the mean [oxy-Hb] changes on the right. The absolute value sign in the formula was suggested by Seghier (2008) to take into account negative values. The laterality index is a ratio measure that reflects differences in the activation level between the two hemispheres in proportion to the overall activation level on both sides, ranging from -1 to 1 . A positive value indicates a left lateralization, whereas a negative value indicates a right lateralization. The laterality index takes into account individuals' overall activation level and allows the comparison of lateralization between groups without being confounded by group differences in activation level. The laterality index has been used in some NIRS studies to investigate lateralization (Chaudhary et al., 2011; Kahlaoui et al., 2012). The 
laterality index was first calculated for each homologous channel pair (e.g., ch1-ch16, ch2-ch14, ch3-ch15, etc.), fluency condition, and participant. Then, we averaged the laterality index across the lateral channel pairs (i.e., ch1-ch16, ch2-ch14, ch3-ch15, and ch4-ch13) and fluency conditions for each participant prior to the statistical analyses. We then tested the mean laterality index against zero using one-sample $t$-tests to investigate lateralization within each group before comparing the MCI and NC groups with an independent sample $t$-test. A similar statistical procedure was repeated for the magnitude of $[\mathrm{oxy}-\mathrm{Hb}$ ] changes to investigate the frontal activation level during the category fluency task within and between the groups.

To further investigate the relationship between the NIRS data (i.e., the laterality index and mean [oxy-Hb] changes) and the behavioral performance of category fluency (i.e., total number of words generated in $60 \mathrm{~s}$ ), Pearson's correlations (two-tailed) were also calculated. All of the statistical analyses were performed using SPSS 22.0 Software (IBM Corporation, Armonk, NY, USA). The significance level was set at 0.05 for all tests.

\section{RESULTS}

\section{Demographic and Neuropsychological Characteristics}

The demographic and neuropsychological characteristics of the MCI and NC groups are presented in Table 2. For the demographic variables, the two groups did not differ significantly in age, education level, gender, or handedness, all $p s>0.10$. For the neuropsychological variables, the MCI group performed significantly worse than the NC group on almost all of the measures $(p s<0.05)$. For memory, the MCI group tended to learn significantly fewer words and recalled significantly fewer words on the HKLLT, and they recalled fewer figures immediately after presentation and after a delay on the WMS-VR than did the NC group. For speed/executive functions, the MCI group was significantly slower on both conditions of the SST and had significantly poorer organization in copying on the RCFT than did the NC group. For language, the MCI group generated significantly fewer unique animal words, $t_{(50)}=2.95, p=0.005, d=0.82$, and transportation words, $t_{(50)}=2.20, p=0.032, d=0.61$, on the category test during the NIRS recording session and had significantly poorer spontaneous naming ability on the BNT than did the NC group. For visuospatial function, the MCI group had significantly poorer accuracy in copying figures both on the WMS-VR and RCFT. Furthermore, the MCI group had a significantly lower CDRS score than did the NC group. However, the MCI and NC groups did not differ significantly in daily and physical functioning levels or depression and anxiety levels (ps > 0.05).

\section{Further Analyses for the Category Fluency Task Performance}

Previous studies have found that the category fluency deficits in individuals with MCI seemed to be mainly attributed to their deficits in generating new subcategories or switching between subcategories (e.g., Bertola et al., 2014), although deficits in creating large clusters might also be a contributing factor (Price et al., 2012). Therefore, we analyzed the underlying cognitive processes, clustering and switching as proposed by Troyer (2000) and new subcategory generation as used by Bertola et al. (2014) during the animal fluency task. The results of the analyses are presented in Table 3. The independent sample $t$-test results showed that the MCI group made significantly fewer switches between subcategories, $t_{(50)}=3.08, p=0.003, d=0.86$, and generated significantly fewer new subcategories than did the NC group, $t_{(50)}=3.93, p<0.001, d=1.09$ (see Table 3). The mean cluster size did not significantly differ between the groups, $t_{(50)}=$ $0.04, p=0.97, d=0.01$. In summary, the results suggest that the reduced animal word generation in the MCI group seemed to be attributed to their impairment in flexible thinking and strategic search and access of semantic information.

The reduced word generation in the MCI group might be simply due to the use of impaired category fluency scores (i.e., language impairment) for MCI classification. To investigate the effect of this, we ran the analyses again after excluding the three participants that were classified into the MCI group solely due to language impairment. None of the results significantly changed. Therefore, individuals with MCI that had objective impairment in non-language domains also had poorer category fluency compared to those with NC. In addition, processing speed may confound the category fluency deficits in the MCI group because the STT-A time was significantly correlated with the total number of animal and transportation words, $r_{(50)}=-0.37, p=0.007$. We ran an Analysis of covariance (ANCOVA) with the STT-A time as the covariate, and the MCI group still generated fewer total unique words than did the NC group, $F_{(1,49)}=6.41, p=0.015, \eta_{p}=0.12$. Thus, processing speed did not seem to be a confounding factor.

\section{NIRS Analysis}

We first investigated the lateralization of oxy-Hb changes using the laterality index, which was averaged over the lateral PFC channel pairs. It should be noted that because the laterality index takes into account the overall activation level on an individual basis, it allows the comparison of the quantity of lateralization between groups without being confounded by group differences in the activation level. A scatter plot of the data is shown in Figure 2. The one-sample $t$-tests showed that the NC group had a significant left lateralization, $t_{(25)}=3.68, p=0.001, d=0.72$,

TABLE 3 | Quantitative analysis of the cognitive processes underlying the category fluency tasks in the $\mathrm{MCl}$ and $\mathrm{NC}$ groups.

\begin{tabular}{llllll}
\hline & \multicolumn{2}{c}{ Group } & & \\
\cline { 2 - 3 } & $\mathbf{M C l}(\boldsymbol{n}=\mathbf{2 6 )}$ & $\mathbf{N C}(\boldsymbol{n}=\mathbf{2 6})$ & $\boldsymbol{t}$ & & $\boldsymbol{p}$ \\
\hline Mean cluster size & $1.06(0.52)$ & $1.07(0.64)$ & 0.04 & 0.97 \\
Number of switches & $6.62(2.50)$ & $9.27(3.61)$ & 3.08 & $0.003^{* * *}$ \\
Number of new subcategories & $5.15(1.71)$ & $7.27(2.15)$ & 3.93 & $<0.001^{* * * *}$ \\
\hline
\end{tabular}

Note: Standard deviations are in parentheses. ${ }^{* *} p<0.01 ;{ }^{* * *} p<0.001$. 


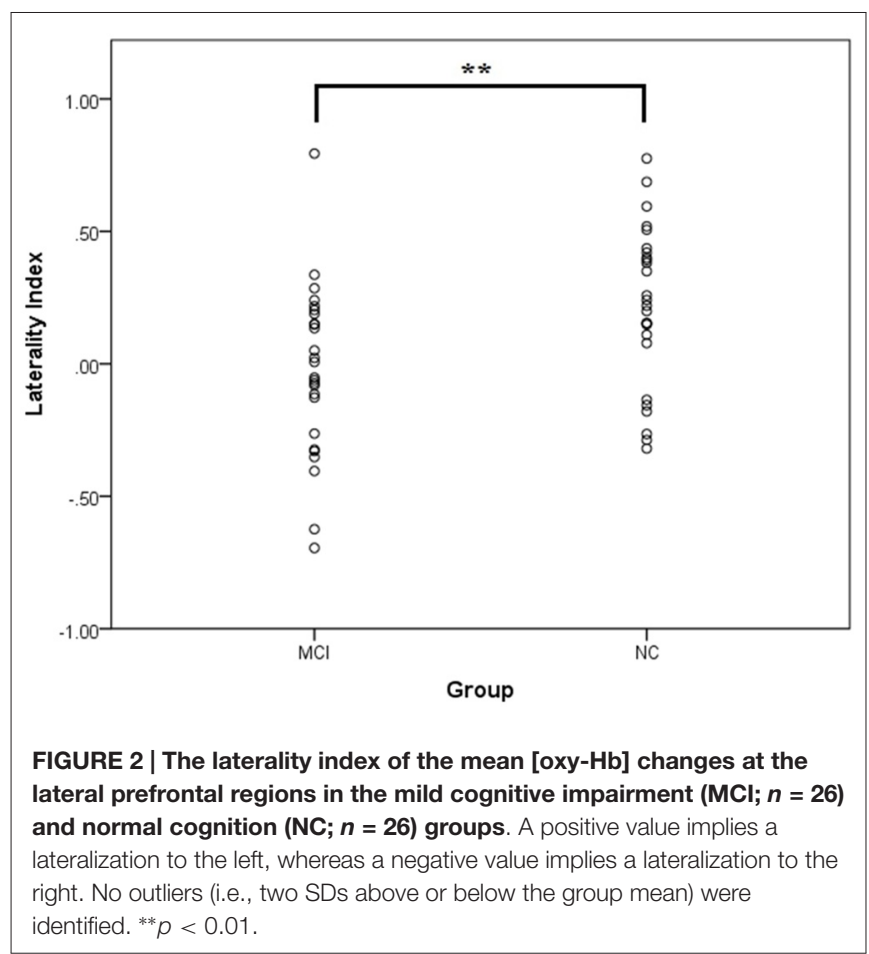

whereas the MCI group did not, $t_{(25)}=0.45, p=0.66, d=0.09$. This result suggests a bilateral activation pattern in the MCI group. An independent samples $t$-test showed that the NC group had a significantly larger laterality index than did the MCI group, $t_{(50)}=2.86, p=0.006, d=0.79$, suggesting that the NC group had a significantly greater left lateralization than did the MCI group. To investigate the confounding effect of the demographic variables, we also performed an ANCOVA with age and education level as the covariates. The group difference in the laterality index remained significant, $F_{(1,48)}=7.14, p=0.010$, $\eta_{p}=0.13$. Thus, the results suggest that compared to the NC group, the MCI group exhibited a reduction and a lack of frontal lateralization during the category fluency task; a finding that was not confounded by group differences in age or education level. The results did not significantly change after excluding the two participants with left handedness, one in each group.

To examine if the two groups showed significant activations on each side during the category fluency task, we analyzed the mean $[\mathrm{oxy}-\mathrm{Hb}$ ] changes within the left and right prefrontal regions. The data are shown in Figure 3. The results of onesample $t$-tests showed that the mean [oxy-Hb] on both sides significantly increased in both the NC group [left: $t_{(25)}=5.14$, $p<0.001, d=1.01$; right: $\left.t_{(25)}=3.61, p=0.001, d=0.71\right]$ and the MCI group [left: $t_{(25)}=4.02, p<0.001, d=0.79$; right: $\left.t_{(25)}=4.43, p<0.001, d=0.87\right]$, suggesting that the lateral prefrontal cortices were significantly activated in both of the groups. Consistent with the results obtained with the laterality index, the MCI group had similar [oxy-Hb] increases on each side, $t_{(25)}=0.39, p=0.70, d=0.05$, whereas the NC group exhibited significantly greater [oxy-Hb] increases on the left than the right, $t_{(25)}=2.38, p=0.025, d=0.29$.

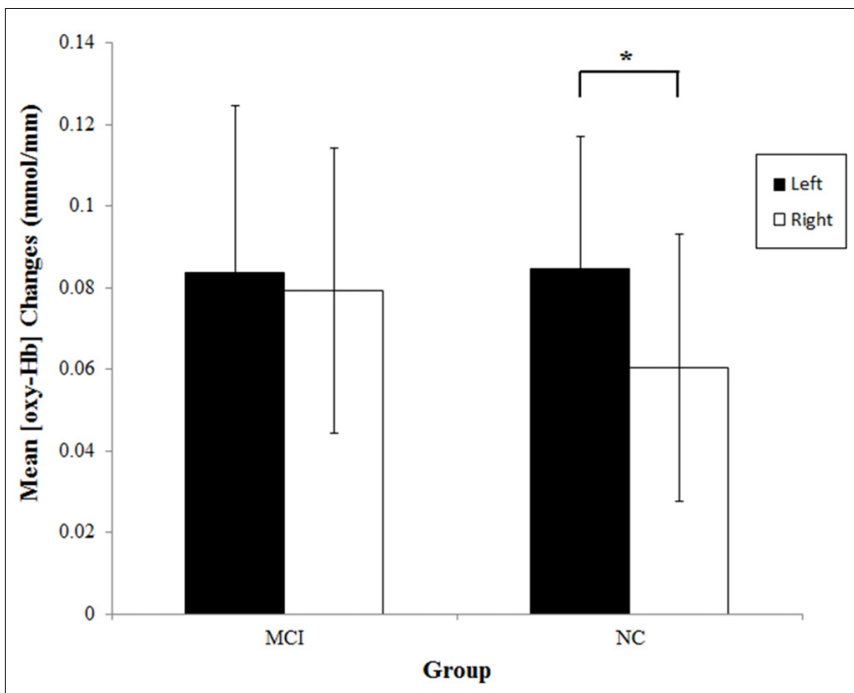

FIGURE 3 | Mean [oxy-Hb] changes at the left (ch13-16) and right (ch1-4) prefrontal regions during the category fluency tasks in the $\mathrm{MCl}$ $(\boldsymbol{n}=\mathbf{2 6})$ and NC $(\boldsymbol{n}=\mathbf{2 6})$ groups. Error bars denote the $95 \%$ confidence interval. ${ }^{*} p<0.05$.

No significant group differences in the mean [oxy-Hb] changes were found [left: $t_{(50)}=0.04, p=0.97, d=0.01$; right: $\left.t_{(50)}=0.77, p=0.45, d=0.21\right]$. None of the results significantly changed after excluding the three participants that were classified into the MCI group solely due to language impairment.

\section{Relationship Between the NIRS Variables, Category Fluency and Neuropsychological Measures}

We first investigated the relationship between the NIRS variables and category fluency performance by calculating Pearson's correlations. The NIRS variables included the left and right [oxy-Hb] changes and laterality index. The results showed that the laterality index was significantly correlated with the total number of unique animal and transportation words generated for the whole group, $r_{(50)}=0.51, p<0.001$. To investigate whether this correlation simply reflected a group difference in the laterality index and task performance, correlation analyses were also performed for each group separately. The results showed that the correlation between the laterality index and category fluency performance was marginally significant within the MCI group, $r_{(24)}=0.38, p=0.056$, and significant within the NC group, $r_{(24)}$ $=0.45, p=0.021$ (see Figure 4). The results did not significantly change after partialling out the effect of age and education level, both for the whole group, $r_{(48)}=0.50, p<0.001$, and separately for the MCI group, $r_{(22)}=0.43, p=0.038$, and the NC group, $r_{(22)}=0.43, p=0.035$. Thus, the results suggest that a greater left lateralization of frontal activations was associated with a better category fluency performance for each of the groups. Neither the [oxy-Hb] changes in the left nor right prefrontal regions were significantly related to the task performance for the whole group, although the direction of the relationship was as expected [left: $r_{(50)}=0.23, p=0.11$; right: $r_{(50)}=-0.04, p=0.79$ ]. 


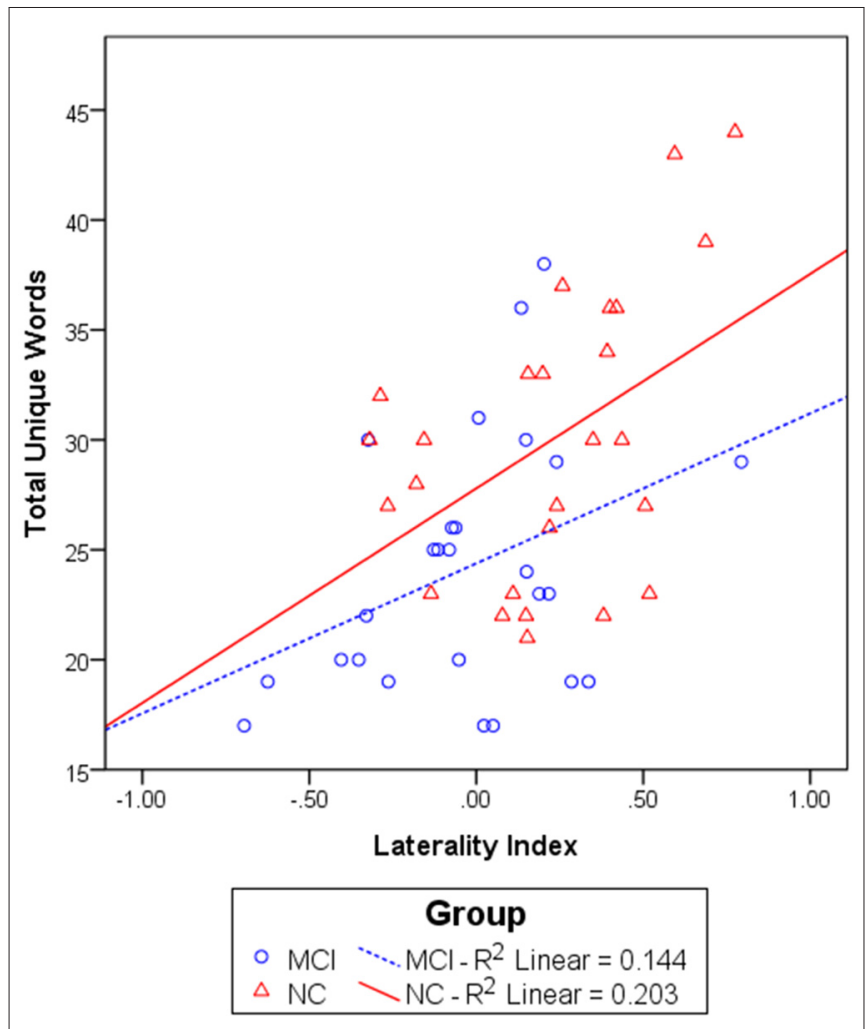

FIGURE 4 | The relationship between the laterality index and total unique words generated in $60 \mathrm{~s}$ in the $\mathrm{MCI}(n=26)$ and $\mathrm{NC}(n=26)$ groups. A linear regression line was fit for each group separately.

Then, to investigate the specificity of the laterality index, we also studied the relationship between the laterality index and other neuropsychological variables. For the neuropsychological variables, one measure was chosen from each cognitive domain to be included for correlational analyses: the delayed recall on the HKLLT for memory, time to completion on trial B of the SST for speed/executive function, spontaneous naming on the BNT for language, and copy score on the RCFT for visuospatial function. The results showed that the laterality index was significantly correlated only with the BNT spontaneous naming score, $r_{(50)}=0.34, p=0.013$. Thus, the laterality index seems to relate and show some specificity with verbal ability.

\section{Subgroup Analyses for Amnestic and Non-Amnestic $\mathrm{MCl}$}

Because different MCI types may be associated with different etiologies (e.g., Yaffe et al., 2006), we repeated the analyses after dividing the MCI group into the amnestic (aMCI; $n=10$ ) and non-amnestic (naMCI; $n=16$ ) groups to investigate if MCI subtype moderated the results. The groups were compared using ANOVA, followed by independent sample $t$-tests if the results were significant. The group differences were not significant for each of the demographic variables, $p$ s $>0.15$, but were significant for almost all of the neuropsychological measures (see Table 4), $p s<0.085$. As expected, the aMCI group had significantly poorer memory (i.e., poorer total learning and delayed recall on the HKLLT and immediate recall on the WMS-VR) compared to the naMCI and TD groups, $p s<0.05$. The group differences in memory between the naMCI and NC groups were not significant, $p s>0.12$. In addition, deficits in speed/executive functions, language, and visuospatial function were more pronounced for the naMCI group and less so for the aMCI group, but the two groups did not significantly differ in these non-memory measures, $p s>0.16$. For the category fluency task (see Table 5), the group differences were significant for the total number of words generated and the number of switches and new subcategories, $p s<0.012$. Both MCI groups generated significantly fewer words compared to the NC group (aMCI: $p=0.012$; naMCI: $p=0.011$ ). In addition, compared to the NC group, both MCI groups generated significantly fewer subcategories (aMCI: $p=0.045$; naMCI: $p<0.001$ ) and made fewer switches (aMCI: $p=0.089$; naMCI: $p=0.007)$. The two MCI groups did not significantly differ, ps $>0.20$.

For the laterality index (see Figure 5), one-sample $t$-tests showed that none of the MCI groups exhibited a significant frontal lateralization [aMCI: $t_{(9)}=0.10, p=0.92, d=0.13$; naMCI: $\left.t_{(15)}=0.74, p=0.47, d=0.18\right]$. ANOVA showed a significant group difference in the laterality index, $F_{(2,49)}=4.36, p=0.018$, $\eta_{p}=0.15$. It was found that compared to the NC group, the naMCI group had a significantly lower laterality index, $t_{(40)}=$ 2.76, $p=0.009, d=0.87$. A tendency for a lower laterality index was also found for the aMCI group, $t_{(34)}=1.72, p=$ $0.095, d=0.59$. The two MCI groups did not significantly differ, $t_{(24)}=0.76, p=0.45, d=0.31$. In addition, all groups exhibited significant $[\mathrm{oxy}-\mathrm{Hb}]$ increases in the left and right prefrontal regions, $p s<0.015$. No group differences were found, $p s>$ 0.30 . None of the MCI groups had significantly greater left than right $[\mathrm{oxy}-\mathrm{Hb}$ ] increases, $\mathrm{ps}>0.32$. In sum, the results suggest that the aMCI and naMCI groups exhibited a similar alteration of frontal activation patterns (e.g., lack of lateralization) during the category fluency task. However, the differences in behavioral performance and neural processing between the MCI and NC groups seem to be driven by the non-amnestic rather than the amnestic subgroups.

\section{DISCUSSION}

In the present study, we examined neural processing during a category fluency task in individuals with MCI by using NIRS. As expected, the behavioral results showed that compared to $\mathrm{NC}$, these individuals generated fewer unique words, made fewer switches between animal subcategories, and generated fewer unique subcategories. In addition, the NIRS results showed that whereas the NC group exhibited a clear left lateralization of frontal activations during the category fluency task, the MCI group did not. A significant and large positive relationship between the category fluency performance and the extent of lateralization of frontal activations was also found. These findings suggest that a reduced lateralization of frontal activations seems to underlie the category fluency deficits in individuals with MCI, especially in those with the non-amnestic subtype. 
TABLE 4 | Demographic, neuropsychological and clinical characteristics of the amnestic (aMCl) and non-amnestic (naMCl) MCl and the NC group.

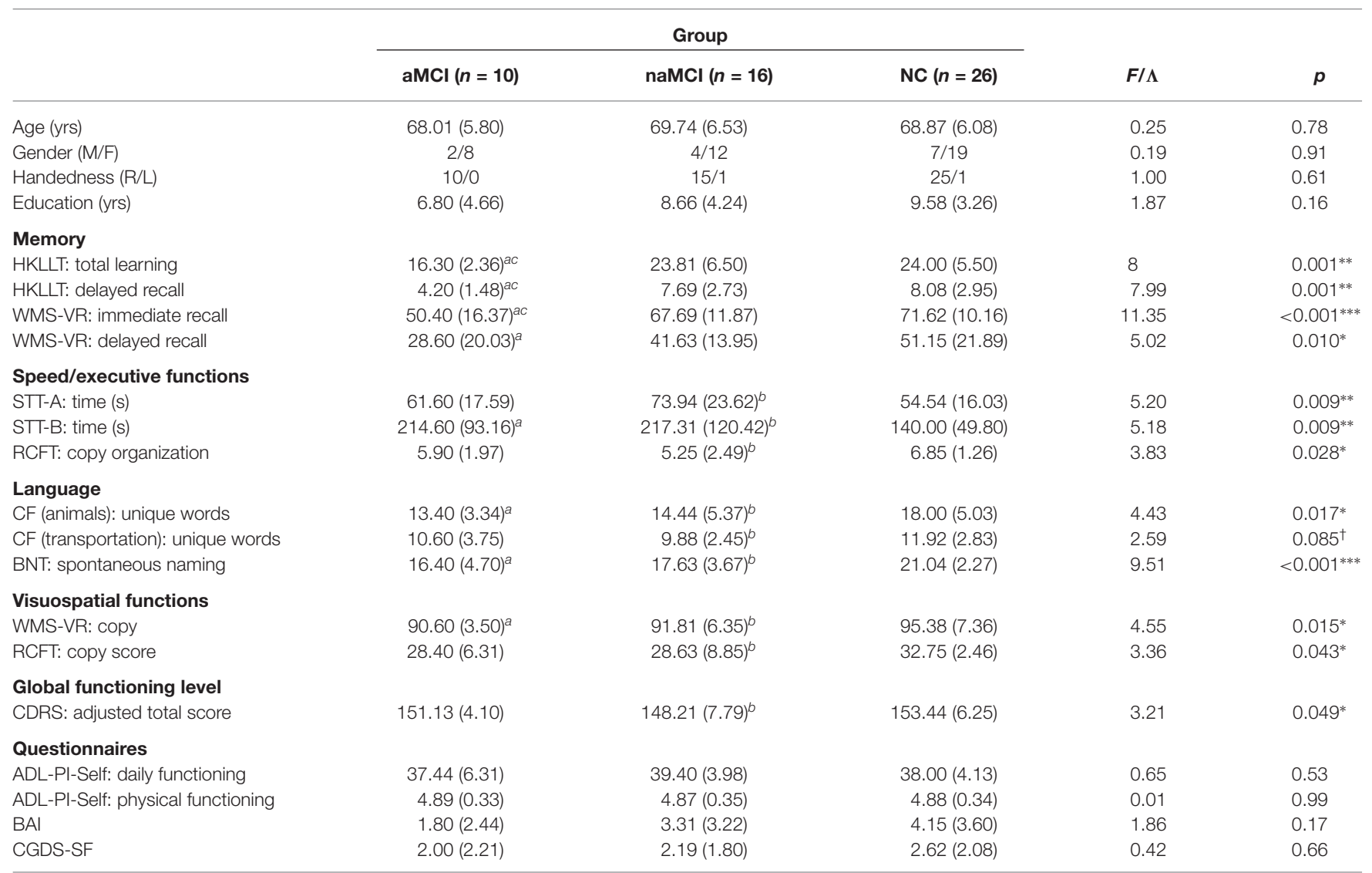

Note: ADL-PI-Self, Self-report version of the Activities of Daily Living-Prevention Instrument; BAI, Beck Anxiety Inventory; BNT, Boston Naming Test; CDRS, Chinese Version of the Mattis Dementia Rating Scale; CF, Category Fluency; CGDS, Chinese Geriatric Depression Scale-Short Form; HKLLT, Hong Kong List Learning Test; RCFT, Rey-Osterrieth Complex Figure Test; ST, Shape Trail Test; WMS-VR, Wechsler Memory Scale-Visual Reproduction. There were four missing values on the ADL-PI-Self (aMCI: $n=1 ;$ naMCl: $n=1 ; N C: n=2$ ), and 1 for the adjusted total score on the CDRS (NC: $n=1$ ). ${ }^{a}$ The aMCl group performed significantly worse than did the NC group, $p<0.05 .{ }^{b}$ The naMCl group performed significantly worse than did the NC group, $p<0.05 .{ }^{c}$ The aMCl group performed significantly worse than did the naMCl group, $p<0.05 .^{\dagger} p<0.10 ;{ }^{*} p<0.05 ; * * 0.01 ; * * *<0.001$.

TABLE 5 | Behavioral performance on the category fluency tasks in the amnestic (aMCl) and non-amnestic (naMCl) MCl and NC groups

\begin{tabular}{|c|c|c|c|c|c|}
\hline & \multicolumn{3}{|c|}{ Group } & $F$ & $p$ \\
\hline Mean cluster size & $0.90(0.59)$ & $1.16(0.52)$ & $1.07(0.64)$ & 0.63 & 0.54 \\
\hline Number of new subcategories & $5.70(1.64)^{a}$ & $4.81(1.72)^{b}$ & $7.27(2.15)$ & 8.41 & $0.001^{* *}$ \\
\hline
\end{tabular}

Note: Standard deviations are in parentheses. ${ }^{a}$ The aMCl group performed significantly worse than did the NC group, $p<0.05 .{ }^{b}$ The naMCl group performed significantly worse than did the NC group, $p<0.05 .{ }^{*} p<0.05 ; * * 0.01$.

Our behavioral findings are consistent with previous findings of word generation deficits in individuals with MCI (Nutter-Upham et al., 2008; Brandt and Manning, 2009; Murphy et al., 2006; Libon et al., 2010; Wang et al., 2011; Price et al., 2012; Bertola et al., 2014; Rinehardt et al., 2014; Vogel et al., 2014; Weakley et al., 2013), suggesting that impaired retrieval of semantic knowledge may already be observed at the earliest stage of transition between normal aging and dementia. Moreover, we found that individuals with MCI generated fewer animal subcategories and make fewer switches between subcategories. This is also in line with the literature that impairment in flexible thinking or searching and accessing novel semantic knowledge may underlie the category fluency deficits in individuals with MCI (Raoux et al., 2008; Price et al., 2012; Weakley et al., 2013; Bertola et al., 2014). On the other hand, individuals with MCI made semantic clusters of 


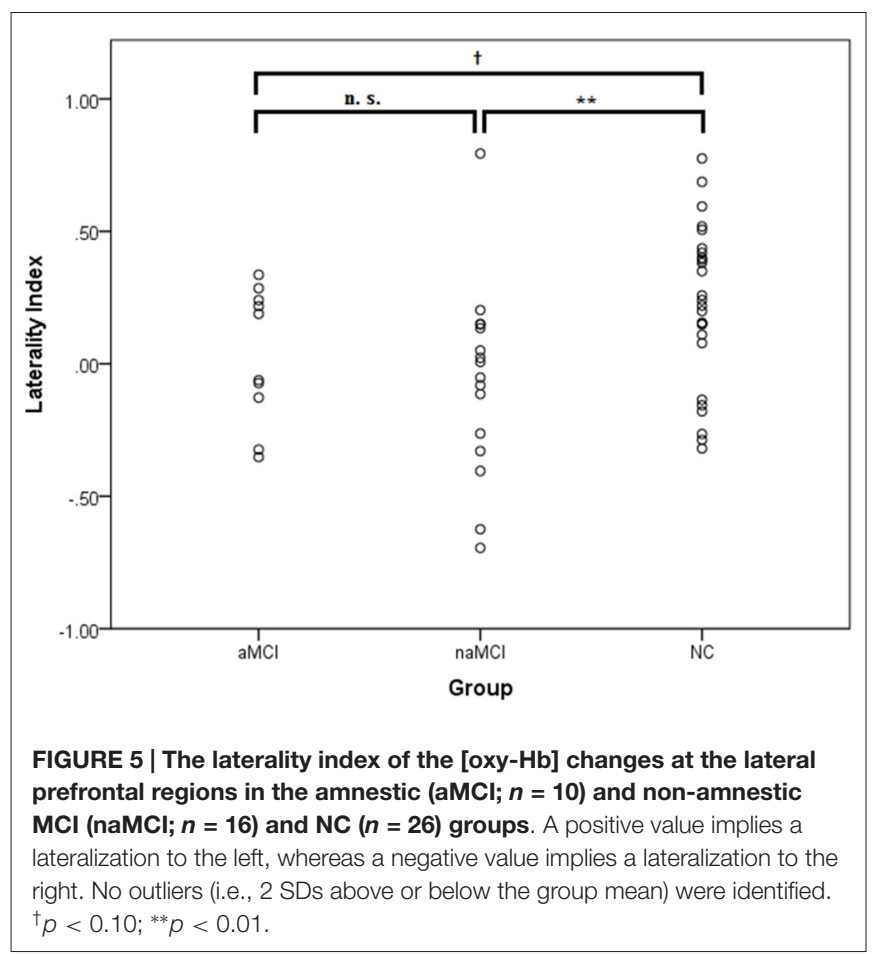

comparable size. This finding is generally consistent with those reported by previous studies (Raoux et al., 2008; Weakley et al., 2013; Bertola et al., 2014; but see Price et al., 2012). We also found that processing speed does not seem to be a confounding factor of the category fluency deficits in MCI, which is in line with a previous study (Price et al., 2012). In sum, our findings are consistent with the literature and confirmed the presence of a category fluency deficit that seems to be attributed to the impairment in flexible thinking and strategic retrieval of semantic knowledge in MCI.

We found that the NC group demonstrated significant [oxy$\mathrm{Hb}$ ] increases in the left and right prefrontal regions, which is consistent with previous findings (Schecklmann et al., 2007; Tupak et al., 2012; Dan et al., 2013; Heinzel et al., 2013, 2015; Liu et al., 2014; Marumo et al., 2014; Holper et al., 2015). In addition, our findings of left-lateralized [oxy-Hb] increases in the NC group are also in line with many of the previous NIRS studies (Fallgatter et al., 1997; Tupak et al., 2012; Dan et al., 2013; Heinzel et al., 2013, 2015), including those that studied older adults (Heinzel et al., 2013, 2015). Our findings of left-lateralized frontal activations during the category fluency task are also consistent with those obtained using other neuroimaging techniques, such as PET (Gourovitch et al., 2000), fMRI (e.g., Weiss et al., 2003; Birn et al., 2010; Wagner et al., 2014), and fTDC (Gutierrez-Sigut et al., 2015). Indeed, the prefrontal cortices, such as the left ventrolateral and dorsolateral prefrontal cortex, have been shown to mediate the strategic retrieval of semantic information (Badre and Wagner, 2007) and flexible thinking (Stuss and Knight, 2013; Yeung et al., 2016) that are demanded by the category fluency task. The frontal activations may reflect the control functions exerted by the frontal lobes during the task performance (Thompson-Schill et al., 1997), and the left lateralization may reflect the verbal nature of the category fluency task (Hellige, 1993). Our findings of correlations between lateralization and the category fluency and naming performance supported the role of lateralization in verbal functioning.

Whereas the NC individuals exhibited a clear pattern of left-lateralized frontal activations, those with MCI in our study did not reveal the same pattern. To our knowledge, the neural processing of individuals with MCI during the category fluency task has not been empirically studied. The present study may serve as a pilot study in this area. Moreover, the present NIRS findings are consistent with previous MRI ( $\mathrm{Pa}$ et al., 2009; Wang et al., 2009; Chang et al., 2010; Ahn et al., 2011; Zhao et al., 2015) and fMRI studies (e.g., Heun et al., 2007; Kaufmann et al., 2008; Alichniewicz et al., 2012; Papma et al., 2013; Van Dam et al., 2013) that found abnormalities in the frontal lobes of individuals with MCI. In addition, some studies found an association between category fluency performance and cortical atrophy of the (left) prefrontal cortex in individuals with MCI (Ahn et al., 2011; Eastman et al., 2013; Clark et al., 2014). Our findings extend the previous literature by showing a relationship between the category fluency performance and on-task prefrontal dynamics. Accordingly, the cortical atrophy and declined functioning of the prefrontal cortex may lead to a change in lateralization during the category fluency task in individuals with MCI. It should be noted that the absence of frontal lateralization during category fluency or other language tasks could been observed in individuals with early left brain injury (Raja Beharelle et al., 2010) or mental disorders and neurological diseases that exhibited category fluency or language deficits, such as Alzheimer's dementia (Fallgatter et al., 1997), schizophrenia (Sommer et al., 2001; Weiss et al., 2004), and developmental dysphasia (de Guibert et al., 2011). Because category fluency is mediated more by the left (frontal) regions (e.g., Wagner et al., 2014), a rightward shift of brain activations in the MCI group may reflect the presence of cortical reorganization in which the contralateral regions (i.e., the right hemisphere) are recruited to serve the function that is declining in the specialized regions (i.e., the left hemisphere). Accordingly, reorganization would not take place if the specialized regions are still well functioning, as in the NC group.

This reorganization account is supported by our strong positive relationship between category fluency performance and the extent of left lateralization, implying a beneficial role of lateralization and a detrimental role of the rightward shift in the task performance. In fact, this finding is quite consistent with the previous findings of an association between poorer category fluency performance and greater right frontal activations or greater left frontal atrophy in older adults with NC (Meinzer et al., 2009) and those with MCI (Eastman et al., 2013; Clark et al., 2014). Because the mean [oxy$\mathrm{Hb}$ ] changes on each side did not significantly correlate with the task performance although they were in the expected direction (left: $r=0.23$; right: $r=-0.04$ ), our study suggests that the relative left-right difference (i.e., the laterality 
index, $r=0.51$ ) may play a more important role than the absolute activation level in determining the category fluency performance. In addition, this brain-behavior relationship was found for both the $\mathrm{NC}$ and MCI groups, suggesting that individuals with $\mathrm{NC}$ and MCI may lie on the same continuum between performance and lateralization, but those with MCI are situated at a lower end of this continuum. Further studies are needed to clarify whether this same relationship might also be found in demented individuals and that demented individuals would show an even more bilateral or right-lateralized activation pattern than did those with MCI.

We further separated the MCI group into the aMCI and naMCI subtypes given that they were associated with different etiologies (Chao et al., 2009; Pa et al., 2009) and dementia outcomes (e.g., Yaffe et al., 2006). That is, the amnestic subtype is more likely to progress to Alzheimer's dementia, while the nonamnestic subtype is more likely to progress to other dementia types (Yaffe et al., 2006). We found that although the two MCI groups did not significantly differ in the category fluency impairment, this impairment was more pronounced in the naMCI group compared to the aMCI group. Our finding is consistent with some previous reports of slightly better category fluency performance in (single-domain) aMCI compared to nonamnestic or dysexecutive MCI (Brandt and Manning, 2009; Libon et al., 2010; Vogel et al., 2014). However, our finding is not consistent with other findings of slightly better category fluency performance in naMCI than aMCI (Weakley et al., 2013; Rinehardt et al., 2014). This discrepancy of findings is possibly attributed to the different composition of the MCI groups (i.e., proportion of the single- and multiple-domain subtypes) in different studies. For example, multiple-domain aMCI has been consistently found to have slightly greater category fluency impairment than single-domain aMCI (Brandt and Manning, 2009; Weakley et al., 2013; Bertola et al., 2014). The mixture of the single- and multiple-domain subtypes of our MCI sample may partly explain the subtly better category fluency performance in the aMCI group compared to the naMCI group in the present study. Altogether, these findings illustrate that the MCI construct is heterogeneous and further research with a large sample of various MCI subtypes (i.e., single-domain, multipledomain, amnestic, non-amnestic) is warranted to verify the level of category fluency impairment associated with each MCI subtype.

In addition, we found that although the aMCI and naMCI groups did not significantly differ in the extent of frontal lateralization during the category fluency task $(p=0.45)$, the frontal abnormality was highly significant in the naMCI group ( $p=0.009$ ) but was only marginally significant in the aMCI group $(p=0.095)$. These findings suggest the presence of more pronounced frontal dysfunction in individuals with naMCI and confirm the heterogeneity of the MCI construct (Petersen et al., 2014). That is, previous studies have found major disturbances in medial temporal lobe structures, such as the hippocampus and entorhinal cortex in aMCI but in other brain regions in naMCI (Chao et al., 2009; Pa et al., 2009). The current findings of frontal dysfunction mainly in the naMCI group are consistent with previous reports of frontal abnormalities that were specific to (dysexecutive) naMCI (Chao et al., 2009; Pa et al., 2009). In addition, although frontal dysfunction is not a major characteristic of patients with aMCI ( $\mathrm{Pa}$ et al., 2009), the tendency for our aMCI group to also exhibit frontal dysfunction may be attributed to an over-representation of individuals with multiple-domain aMCI that had executive dysfunction (i.e., significantly larger STT-B: time) in addition to memory deficits. Alternatively, it is possible that disturbances in medial temporal lobe structures may alter the normal functioning of the prefrontal cortex during cognitive tasks since the medial temporal lobe is closely connected with the frontal lobe (Simons and Spiers, 2003). Given these speculations and the heterogeneity of the MCI construct, further studies with a large sample of individuals with singledomain aMCI (i.e., pure memory problems) may help explain the observed tendency for frontal dysfunction in our aMCI group.

Our findings have several important implications. First, we found that individuals with MCI did not exhibit a lateralization of frontal activations during the category fluency task. This finding suggests that the lateralization pattern may serve as a neural marker for distinguishing between normal aging and MCI. Developing and identifying objective markers of MCI is important because early detection of patients at the prodromal stage of dementia offers the best potential for intervention (Ernst et al., 1997). Given that NIRS is time- and cost-effective and easy to administer, it seems to be a promising tool for probing the neural functioning in various clinical populations. In addition, our lateralization finding may also serve as a neural marker for the evaluation of the effectiveness of interventions that target the MCI population in the future. Furthermore, our study showed that the NC and MCI were on the same continuum between the category fluency performance and lateralization, suggesting that cognitively normal older adults that exhibited both a low category fluency score and a small extent of lateralization may have increased risks for the development of MCI or dementia. To verify this speculation, longitudinal studies that track cognitive changes in cognitively normal older adults that exhibited a large or a small lateralization are needed.

Although the present study has enhanced our understanding of the neural basis of the category fluency deficits in individuals with MCI, some limitations must be mentioned. First, the NIRS measurement was restricted to the prefrontal regions. Given that the category fluency performance may engage non-frontal regions (Birn et al., 2010; Wagner et al., 2014), the MCI group might have altered activations in non-frontal regions. In addition, we primarily combined the amnestic and non-amnestic MCI subtypes into one single MCI group because of the non-significant differences between the two and the relatively small sample size. Some studies reported that the amnestic subtype was more likely to progress to Alzheimer's dementia, while the non-amnestic subtype was more likely to progress to other dementia types (e.g., Yaffe et al., 2006), suggesting different etiologies between the two subtypes. Further studies with a larger sample are needed 
to clarify if the two MCI subtypes would differ in the neural processing during the category fluency task and if the lateralization pattern can reliably predict specific forms of dementia. Furthermore, because structural brain images were not acquired, the relationship between lateralization and brain structure remains to be determined. Moreover, although we demonstrated that education was not a confounding factor of the present findings, education was not closely matched between the groups. Moreover, females were over-represented in the sample. Thus, further investigations with a larger sample that has a more even gender distribution and a closer match on education are warranted.

In conclusion, we found that individuals with MCI had category fluency deficits and that impairment in flexible thinking and strategic search and access of semantic information seem to underlie these deficits. In addition, we extend the literature by providing the neural basis of the category fluency deficits in these individuals, showing that they did not exhibit a leftlateralization of frontal activations, unlike their cognitively normal counterparts, during the category fluency task. This rightward shift of activations may reflect the presence of cortical reorganization, in which the contralateral hemispheric takes over the function that is declining in the specialized hemisphere. This interpretation is supported by the finding of a strong relationship between the lack of lateralization and category fluency deficits. To verify the potentiality of the use of NIRS for clinical diagnosis and the evaluation of the effectiveness of interventions that target the MCI population, further studies are needed. Our findings suggest that a neural disturbance during the category fluency task may already be present at the prodromal

\section{REFERENCES}

Ahn, H., Seo, S. W., Chin, J., Suh, M. K., Lee, B. H., Kim, S. T., et al. (2011). The cortical neuroanatomy of neuropsychological deficits in mild cognitive impairment and Alzheimer's disease: a surface-based morphometric analysis. Neuropsychologia 49, 3931-3945. doi: 10.1016/j.neuropsychologia.2011. 10.010

Albert, M. S., DeKosky, S. T., Dickson, D., Dubois, B., Feldman, H. H., Fox, N. C., et al. (2011). The diagnosis of mild cognitive impairment due to Alzheimer's disease: recommendations from the national institute on aging-Alzheimer's association workgroups on diagnostic guidelines for Alzheimer's disease. Alzheimers Dement. 7, 270-279. doi: 10.1016/j.jalz.2011. 03.008

Alichniewicz, K. K., Brunner, F., Klünemann, H. H., and Greenlee, M. W. (2012). Structural and functional neural correlates of visuospatial information processing in normal aging and amnestic mild cognitive impairment. Neurobiol. Aging 33, 2782-2797. doi: 10.1016/j.neurobiolaging.2012. 02.010

Amieva, H., Letenneur, L., Dartigues, J. F., Rouch-Leroyer, I., Sourgen, C., D’Alchée-Birée, F., et al. (2004). Annual rate and predictors of conversion to dementia in subjects presenting mild cognitive impairment criteria defined according to a population-based study. Dement. Geriatr. Cogn. Disord. 18, 87-93. doi: 10.1159/000077815

Badre, D., and Wagner, A. D. (2007). Left ventrolateral prefrontal cortex and the cognitive control of memory. Neuropsychologia 45, 2883-2901. doi: 10.1016/j. neuropsychologia.2007.06.015

Beck, A. T., Epstein, N., Brown, G., and Steer, R. A. (1988). An inventory for measuring clinical anxiety: psychometric properties. J. Consult. Clin. Psychol. 56, 893-897. doi: 10.1037/0022-006x.56.6.893 stage of dementia. In addition, given that the category fluency impairment and frontal abnormalities were highly significant in the naMCI group but only marginally significant in the aMCI group, our study also highlights the heterogeneity of the MCI construct.

\section{AUTHOR CONTRIBUTIONS}

ASC, SLS, JW, TK, DHKS, and RY conceived the study. All authors led the design of the experiment. SLS and RY led the field implementation. MKY, SLS, and RY led the data collection. MKY prepared the data for analyses. MKY and ASC engaged in the analyses. MKY, ASC, JW, TK, DHKS, RY contributed to the interpretation of the data. MKY and ASC drafted the first manuscript. All authors work on the revisions of the drafts, critically contributed to its content and approved the final version of the manuscript.

\section{ACKNOWLEDGMENTS}

This work was supported by the Social Science Collaborative Research Fund (SSCRF) from the Faculty of Social Science of the Chinese University of Hong Kong. The authors would like to specially thank Winnie Cheung, Lan He, Jia-yin Huang, Chloe $\mathrm{Ng}$, Leo Ng, Victoria Tang, Rex Wang, and Debbie Yan for their effort in subject recruitment, data collection and management. They would also like to thank Chun-yu Tse for providing feedbacks to the manuscript. Further appreciation is extended to all participants and to the community health and social centers for their assistance in the recruitment process.

Bernstein, J. H., and Waber, D. P. (1996). Developmental Scoring System for the Rey-Osterrieth Complex Figure. Odessa, FL: Psychological Assessment Resources.

Bertola, L., Lima, M. L. C., Romano-Silva, M. A., de Moraes, E. N., Diniz, B. S., and Malloy-Diniz, L. F. (2014). Impaired generation of new subcategories and switching in a semantic verbal fluency test in older adults with mild cognitive impairment. Front. Aging Neurosci. 6:141. doi: 10.3389/fnagi.2014. 00141

Birn, R. M., Kenworthy, L., Case, L., Caravella, R., Jones, T. B., Bandettini, P. A., et al. (2010). Neural systems supporting lexical search guided by letter and semantic category cues: a self-paced overt response fMRI study of verbal fluency. Neuroimage 49, 1099-1107. doi: 10.1016/j.neuroimage.2009. 07.036

Bondi, M. W., Edmonds, E. C., Jak, A. J., Clark, L. R., Delano-Wood, L., McDonald, C. R., et al. (2014). Neuropsychological criteria for mild cognitive impairment improves diagnostic precision, biomarker associations and progression rates. J. Alzheimers Dis. 42, 275-289. doi: 10.3233/JAD140276

Brandt, J., and Manning, K. J. (2009). Patterns of word-list generation in mild cognitive impairment and Alzheimer's disease. Clin. Neuropsychol. 23, 870-879. doi: 10.1080/138540408025 85063

Chan, A. S., Choi, M. K., and Salmon, D. P. (2001). The effects of age, education and gender on the mattis dementia rating scale performance of elderly chinese and american individuals. J. Gerontol. B Psychol. Sci. Soc. Sci. 56, P356-P363. doi: 10.1093/geronb/56.6.p356

Chan, A. S., and Kwok, I. (2006). Hong Kong List Learning Test. 2nd Edn. Hong Kong: Department of Psychological and Integrative Neuropsychological Rehabilitation Center. 
Chan, A. S., and Poon, M. W. (1999). Performance of 7-to 95-year-old individuals in a chinese version of the category fluency test. J. Int. Neuropsychol. Soc. 5, 525-533. doi: 10.1017/s135561779956606x

Chang, Y.-L., Jacobson, M. W., Fennema-Notestine, C., Hagler, D. J., Jr., Jennings, R. G., Dale, A. M., et al. (2010). Level of executive function influences verbal memory in amnestic mild cognitive impairment and predicts prefrontal and posterior cingulate thickness. Cereb. Cortex 20, 1305-1313. doi: 10. 1093/cercor/bhp192

Chao, L. L., Pa, J., Duarte, A., Schuff, N., Weiner, M. W., Kramer, J. H., et al. (2009). Patterns of cerebral hypoperfusion in amnestic and dysexecutive MCI. Alzheimer Dis. Assoc. Disord. 23, 245-252. doi: 10.1097/WAD. 0b013e318199ff46

Chaudhary, U., Hall, M., DeCerce, J., Rey, G., and Godavarty, A. (2011). Frontal activation and connectivity using near-infrared spectroscopy: verbal fluency language study. Brain Res. Bull. 84, 197-205. doi: 10.1016/j.brainresbull.2011. 01.002

Cheung, R. W., Cheung, M., and Chan, A. S. (2004). Confrontation naming in chinese patients with left, right or bilateral brain damage. J. Int. Neuropsychol. Soc. 10, 46-53. doi: 10.1017/s1355617704101069

Clark, L. R., Delano-Wood, L., Libon, D. J., McDonald, C. R., Nation, D. A., Bangen, K. J., et al. (2013). Are empirically-derived subtypes of mild cognitive impairment consistent with conventional subtypes? J. Int. Neuropsychol. Soc. 19, 635-645. doi: 10.1017/s1355617713000313

Clark, D., Wadley, V., Kapur, P., DeRamus, T., Singletary, B., Nicholas, A., et al. (2014). Lexical factors and cerebral regions influencing verbal fluency performance in MCI. Neuropsychologia 54, 98-111. doi: 10.1016/j. neuropsychologia.2013.12.010

Cui, X., Bray, S., Bryant, D. M., Glover, G. H., and Reiss, A. L. (2011). A quantitative comparison of NIRS and fMRI across multiple cognitive tasks. Neuroimage 54, 2808-2821. doi: 10.1016/j.neuroimage.2010. 10.069

Dan, H., Dan, I., Sano, T., Kyutoku, Y., Oguro, K., Yokota, H., et al. (2013). Language-specific cortical activation patterns for verbal fluency tasks in japanese as assessed by multichannel functional near-infrared spectroscopy. Brain Lang. 126, 208-216. doi: 10.1016/j.bandl.2013. 05.007

Dannhauser, T. M., Walker, Z., Stevens, T., Lee, L., Seal, M., and Shergill, S. S. (2005). The functional anatomy of divided attention in amnestic mild cognitive impairment. Brain 128, 1418-1427. doi: 10.1093/brain/awh413

de Guibert, C., Maumet, C., Jannin, P., Ferré, J., Tréguier, C., Barillot, C., et al. (2011). Abnormal functional lateralization and activity of language brain areas in typical specific language impairment (developmental dysphasia). Brain 134, 3044-3058. doi: 10.1093/brain/awr141

Delpy, D. T., Cope, M., van der Zee, P., Arridge, S. R., Wray, S., and Wyatt, J. S. (1988). Estimation of optical pathlength through tissue from direct time of flight measurement. Phys. Med. Biol. 33, 1433-1442. doi: 10.1088/00319155/33/12/008

Eastman, J. A., Hwang, K. S., Lazaris, A., Chow, N., Ramirez, L., Babakchanian, S., et al. (2013). Cortical thickness and semantic fluency in Alzheimer's disease and mild cognitive impairment. Am. J. Alzheimers Dis. (Columbia) 1, 81-92. doi: 10. 7726/ajad.2013.1006

Ehlis, A., Herrmann, M. J., Plichta, M. M., and Fallgatter, A. J. (2007). Cortical activation during two verbal fluency tasks in schizophrenic patients and healthy controls as assessed by multi-channel near-infrared spectroscopy. Psychiatry Res. 156, 1-13. doi: 10.1016/j.pscychresns.2006.11.007

Ernst, R. L., Hay, J. W., Fenn, C., Tinklenberg, J., and Yesavage, J. A. (1997). Cognitive function and the costs of Alzheimer disease: an exploratory study. Arch. Neurol. 54, 687-693. doi: 10.1001/archneur.1997.005501800 13006

Fallgatter, A., Roesler, M., Sitzmann, A., Heidrich, A., Mueller, T., and Strik, W. (1997). Loss of functional hemispheric asymmetry in Alzheimer's dementia assessed with near-infrared spectroscopy. Brain Res. Cogn. Brain Res. 6, 67-72. doi: 10.1016/s0926-6410(97)00016-5

Galasko, D., Bennett, D. A., Sano, M., Marson, D., Kaye, J., and Edland, S. D. (2006). ADCS prevention instrument project: assessment of instrumental activities of daily living for community-dwelling elderly individuals in dementia prevention clinical trials. Alzheimer Dis. Assoc. Disord. 20, S152-S169. doi: 10.1097/01.wad.0000213873.25053.2b
Gourovitch, M. L., Kirkby, B. S., Goldberg, T. E., Weinberger, D. R., Gold, J. M., Esposito, G., et al. (2000). A comparison of rCBF patterns during letter and semantic fluency. Neuropsychology 14, 353-360. doi: 10.1037/0894-4105. 14.3.353

Gutierrez-Sigut, E., Payne, H., and MacSweeney, M. (2015). Investigating language lateralization during phonological and semantic fluency tasks using functional transcranial doppler sonography. Laterality 20, 49-68. doi: 10.1080/1357650x. 2014.914950

Heinzel, S., Metzger, F. G., Ehlis, A., Korell, R., Alboji, A., Haeussinger, F. B., et al. (2013). Aging-related cortical reorganization of verbal fluency processing: a functional near-infrared spectroscopy study. Neurobiol. Aging 34, 439-450. doi: 10.1016/j.neurobiolaging.2012.05.021

Heinzel, S., Metzger, F. G., Ehlis, A., Korell, R., Alboji, A., Haeussinger, F. B., et al. (2015). Age and vascular burden determinants of cortical hemodynamics underlying verbal fluency. PLoS One 10:e0138863. doi: 10.1371/journal.pone. 0138863

Hellige, J. B. (1993). Hemispheric Asymmetry: What's Right and What's Left. Cambridge, MA: Harvard University Press.

Heun, R., Freymann, K., Erb, M., Leube, D. T., Jessen, F., Kircher, T. T., et al. (2007). Mild cognitive impairment (MCI) and actual retrieval performance affect cerebral activation in the elderly. Neurobiol. Aging 28, 404-413. doi: 10. 1016/j.neurobiolaging.2006.01.012

Holper, L., Aleksandrowicz, A., Müller, M., Ajdacic-Gross, V., Haker, H., Fallgatter, A., et al. (2015). Brain correlates of verbal fluency in subthreshold psychosis assessed by functional near-infrared spectroscopy. Schizophr. Res. 168, 23-29. doi: 10.1016/j.schres.2015.07.043

Hori, H., Ozeki, Y., Terada, S., and Kunugi, H. (2008). Functional near-infrared spectroscopy reveals altered hemispheric laterality in relation to schizotypy during verbal fluency task. Prog. Neuropsychopharmacol. Biol. Psychiatry 32, 1944-1951. doi: 10.1016/j.pnpbp.2008.09.019

Jak, A. J., Bondi, M. W., Delano-Wood, L., Wierenga, C., Corey-Bloom, J., Salmon, D. P., et al. (2009). Quantification of five neuropsychological approaches to defining mild cognitive impairment. Am. J. Geriatr. Psychiatry 17, 368-375. doi: 10.1097/JGP.0b013e31819431d5

Jasper, H. H. (1958). The 10/20 international electrode system. Electroencephalogr. Clin. Neurophysiol. 10, 371-375.

Kahlaoui, K., Di Sante, G., Barbeau, J., Maheux, M., Lesage, F., Ska, B., et al. (2012). Contribution of NIRS to the study of prefrontal cortex for verbal fluency in aging. Brain Lang. 121, 164-173. doi: 10.1016/j.bandl.2011. 11.002

Kaufmann, L., Ischebeck, A., Weiss, E., Koppelstaetter, F., Siedentopf, C., Vogel, S. E., et al. (2008). An fMRI study of the numerical stroop task in individuals with and without minimal cognitive impairment. Cortex 44, 1248-1255. doi: 10 1016/j.cortex.2007.11.009

Kubota, Y., Toichi, M., Shimizu, M., Mason, R. A., Coconcea, C. M., Findling, R. L., et al. (2005). Prefrontal activation during verbal fluency tests in schizophrenia-a near-infrared spectroscopy (NIRS) study. Schizophr. Res. 77, 65-73. doi: 10.1016/j.schres.2005.01.007

Lee, H. B., Chiu, H. F., Kowk, W. Y., and Leung, C. M. (1993). Chinese elderly and the GDS short form: a preliminary study. Clin. Gerontol. J. Aging Ment. Health $14,37-42$.

Li, H., Hou, X., Liu, H., Yue, C., He, Y., and Zuo, X. (2015). Toward systems neuroscience in mild cognitive impairment and Alzheimer's disease: a metaanalysis of 75 fMRI studies. Hum. Brain Mapp. 36, 1217-1232. doi: 10. 1002/hbm.22689

Libon, D. J., Xie, S. X., Eppig, J., Wicas, G., Lamar, M., Lippa, C., et al. (2010). The heterogeneity of mild cognitive impairment: a neuropsychological analysis. J. Int. Neuropsychol. Soc. 16, 84-93. doi: 10.1017/s1355617709 990993

Liu, X., Sun, G., Zhang, X., Xu, B., Shen, C., Shi, L., et al. (2014). Relationship between the prefrontal function and the severity of the emotional symptoms during a verbal fluency task in patients with major depressive disorder: a multi-channel NIRS study. Prog. Neuropsychopharmacol. Biol. Psychiatry 54, 114-121. doi: 10.1016/j.pnpbp.2014. 05.005

Makizako, H., Doi, T., Shimada, H., Park, H., Uemura, K., Yoshida, D., et al. (2013). Relationship between going outdoors daily and activation of the prefrontal cortex during verbal fluency tasks (VFTs) among older adults: a 
near-infrared spectroscopy study. Arch. Gerontol. Geriatr. 56, 118-123. doi: 10. 1016/j.archger.2012.08.017

Marumo, K., Takizawa, R., Kinou, M., Kawasaki, S., Kawakubo, Y., Fukuda, M., et al. (2014). Functional abnormalities in the left ventrolateral prefrontal cortex during a semantic fluency task and their association with thought disorder in patients with schizophrenia. Neuroimage 85, 518-526. doi: 10 . 1016/j.neuroimage.2013.04.050

Meinzer, M., Flaisch, T., Wilser, L., Eulitz, C., Rockstroh, B., Conway, T., et al. (2009). Neural signatures of semantic and phonemic fluency in young and old adults. J. Cogn. Neurosci. 21, 2007-2018. doi: 10.1162/jocn.2009. 21219

Meinzer, M., Lindenberg, R., Antonenko, D., Flaisch, T., and Floel, A. (2013). Anodal transcranial direct current stimulation temporarily reverses ageassociated cognitive decline and functional brain activity changes. J. Neurosci. 33, 12470-12478. doi: 10.1523/JNEUROSCI.5743-12.2013

Molinuevo, J. L., Gómez-Anson, B., Monte, G. C., Bosch, B., Sánchez-Valle, R., and Rami, L. (2011). Neuropsychological profile of prodromal Alzheimer's disease (prd-AD) and their radiological correlates. Arch. Gerontol. Geriatr. 52, 190-196. doi: 10.1016/j.archger.2010.03.016

Murphy, K. J., Rich, J. B., and Troyer, A. K. (2006). Verbal fluency patterns in amnestic mild cognitive impairment are characteristic of Alzheimer's type dementia. J. Int. Neuropsychol. Soc. 12, 570-574. doi: 10. $1017 /$ s1355617706060590

Nutter-Upham, K. E., Saykin, A. J., Rabin, L. A., Roth, R. M., Wishart, H. A., Pare, N., et al. (2008). Verbal fluency performance in amnestic MCI and older adults with cognitive complaints. Arch. Clin. Neuropsychol. 23, 229-241. doi: 10.1016/j.acn.2008.01.005

Pa, J., Boxer, A., Chao, L. L., Gazzaley, A., Freeman, K., Kramer, J., et al. (2009). Clinical-neuroimaging characteristics of dysexecutive mild cognitive impairment. Ann. Neurol. 65, 414-423. doi: 10.1002/ana.21591

Papma, J. M., den Heijer, T., de Koning, I., Mattace-Raso, F. U., van der Lugt, A., van der Lijn, F., et al. (2013). The influence of cerebral small vessel disease on default mode network deactivation in mild cognitive impairment. Neuroimage Clin. 2, 33-42. doi: 10.1016/j.nicl.2012.11.005

Petersen, R., Caracciolo, B., Brayne, C., Gauthier, S., Jelic, V., and Fratiglioni, L. (2014). Mild cognitive impairment: a concept in evolution. J. Intern. Med. 275, 214-228. doi: 10.1111/joim. 12190

Petersen, R. C., Smith, G. E., Waring, S. C., Ivnik, R. J., Tangalos, E. G., and Kokmen, E. (1999). Mild cognitive impairment: clinical characterization and outcome. Arch. Neurol. 56, 303-308. doi: 10.1001/archneur.56.3.303

Price, S. E., Kinsella, G. J., Ong, B., Storey, E., Mullaly, E., Phillips, M., et al. (2012). Semantic verbal fluency strategies in amnestic mild cognitive impairment. Neuropsychology 26, 490-497. doi: 10.1037/a0028567

Raja Beharelle, A., Dick, A. S., Josse, G., Solodkin, A., Huttenlocher, P. R., Levine, S. C., et al. (2010). Left hemisphere regions are critical for language in the face of early left focal brain injury. Brain 133, 1707-1716. doi: 10.1093/brain/ awQ114

Raoux, N., Amieva, H., Le Goff, M., Auriacombe, S., Carcaillon, L., Letenneur, L., et al. (2008). Clustering and switching processes in semantic verbal fluency in the course of Alzheimer's disease subjects: results from the PAQUID longitudinal study. Cortex 44, 1188-1196. doi: 10.1016/j.cortex.2007.08.019

Rinehardt, E., Eichstaedt, K., Schinka, J. A., Loewenstein, D. A., Mattingly, M., Fils, J., et al. (2014). Verbal fluency patterns in mild cognitive impairment and Alzheimer's disease. Dement. Geriatr. Cogn. Disord. 38, 1-9. doi: 10. $1159 / 000355558$

Sato, H., Yahata, N., Funane, T., Takizawa, R., Katura, T., Atsumori, H., et al. (2013). A NIRS-fMRI investigation of prefrontal cortex activity during a working memory task. Neuroimage 83, 158-173. doi: 10.1016/j.neuroimage. 2013.06.043

Schecklmann, M., Ehlis, A., Plichta, M. M., Boutter, H. K., Metzger, F. G., and Fallgatter, A. J. (2007). Altered frontal brain oxygenation in detoxified alcohol dependent patients with unaffected verbal fluency performance. Psychiatry Res. 156, 129-138. doi: 10.1016/j.pscychresns.2007.01.009

Seghier, M. L. (2008). Laterality index in functional MRI: methodological issues. Magn. Reson. Imaging 26, 594-601. doi: 10.1016/j.mri.2007.10.010

Simons, J. S., and Spiers, H. J. (2003). Prefrontal and medial temporal lobe interactions in long-term memory. Nat. Rev. Neurosci. 4, 637-648. doi: 10. 1038/nrn1178
Sommer, I. E. C., Ramsey, N. F., and Kahn, R. S. (2001). Language lateralization in schizophrenia, an fMRI study. Schizophr. Res. 52, 57-67. doi: 10.1016/s09209964(00)00180-8

Strangman, G., Culver, J. P., Thompson, J. H., and Boas, D. A. (2002). A quantitative comparison of simultaneous BOLD fMRI and NIRS recordings during functional brain activation. Neuroimage 17, 719-731. doi: 10 1006/nimg.2002.1227

Stuss, D. T., and Knight, R. T. (2013). Principles of Frontal Lobe Function. New York, NY: Oxford University Press.

Thompson-Schill, S. L., D’Esposito, M., Aguirre, G. K., and Farah, M. J. (1997). Role of left inferior prefrontal cortex in retrieval of semantic knowledge: a reevaluation. Proc. Natl. Acad. Sci. U S A 94, 14792-14797. doi: 10.1073/pnas. 94.26.14792

Tierney, M. C., Yao, C., Kiss, A., and McDowell, I. (2005). Neuropsychological tests accurately predict incident Alzheimer disease after 5 and 10 years. Neurology 64, 1853-1859. doi: 10.1212/01.WNL.0000163773.217 $94.0 \mathrm{~B}$

Troyer, A. K. (2000). Normative data for clustering and switching on verbal fluency tasks. J. Clin. Exp. Neuropsychol. 22, 370-378. doi: 10.1076/13803395(200006)22:3;1-v;ft370

Tupak, S. V., Badewien, M., Dresler, T., Hahn, T., Ernst, L. H., Herrmann, M. J., et al. (2012). Differential prefrontal and frontotemporal oxygenation patterns during phonemic and semantic verbal fluency. Neuropsychologia 50, 1565-1569. doi: 10.1016/j.neuropsychologia.2012. 03.009

Van Dam, N. T., Sano, M., Mitsis, E. M., Grossman, H. T., Gu, X., Park, Y., et al. (2013). Functional neural correlates of attentional deficits in amnestic mild cognitive impairment. PLoS One 8:e54035. doi: 10.1371/journal.pone. 0054035

Venneri, A., Gorgoglione, G., Toraci, C., Nocetti, L., Panzetti, P., and Nichelli, P. (2011). Combining neuropsychological and structural neuroimaging indicators of conversion to Alzheimer's disease in amnestic mild cognitive impairment. Curr. Alzheimer Res. 8, 789-797. doi: 10.2174/156720511797 633160

Villringer, A., and Chance, B. (1997). Non-invasive optical spectroscopy and imaging of human brain function. Trends Neurosci. 20, 435-442. doi: 10. 1016/S0166-2236(97)01132-6

Vogel, A., Johannsen, P., Stokholm, J., and Jørgensen, K. (2014). Frequency and severity of semantic deficits in a consecutive memory clinic cohort. Dement. Geriatr. Cogn. Disord. 38, 214-223. doi: 10.1159/000357794

Wagner, S., Sebastian, A., Lieb, K., Tüscher, O., and Tadić, A. (2014). A coordinate-based ALE functional MRI meta-analysis of brain activation during verbal fluency tasks in healthy control subjects. BMC Neurosci. 15:19. doi: 10 . 1186/1471-2202-15-19

Wang, L., Goldstein, F. C., Veledar, E., Levey, A. I., Lah, J. J., Meltzer, C. C., et al. (2009). Alterations in cortical thickness and white matter integrity in mild cognitive impairment measured by whole-brain cortical thickness mapping and diffusion tensor imaging. AJNR Am. J. Neuroradiol. 30, 893-899. doi: 10 . 3174/ajnr.a1484

Wang, P.-N., Hong, C.-J., Lin, K.-N., Liu, H.-C., and Chen, W.-T. (2011). APOE $\varepsilon 4$ increases the risk of progression from amnestic mild cognitive impairment to Alzheimer's disease among ethnic chinese in taiwan. J. Neurol Neurosurg. Psychiatry 82, 165-169. doi: 10.1136/jnnp.2010.209122

Weakley, A., Schmitter-Edgecombe, M., and Anderson, J. (2013). Analysis of verbal fluency ability in amnestic and non-amnestic mild cognitive impairment. Arch. Clin. Neuropsychol. 28, 721-731. doi: 10.1093/arclin/act058

Wechsler, D. (2005). Wechsler Memory Scale III (Chinese): Manual. Taiwan: Psychological Corporation.

Weiss, E. M., Hofer, A., Golaszewski, S., Siedentopf, C., Brinkhoff, C., Kremser, C., et al. (2004). Brain activation patterns during a verbal fluency test-a functional MRI study in healthy volunteers and patients with schizophrenia. Schizophr. Res. 70, 287-291. doi: 10.1016/j.schres.2004.01.010

Weiss, E., Siedentopf, C., Hofer, A., Deisenhammer, E., Hoptman, M., Kremser, C. et al. (2003). Brain activation pattern during a verbal fluency test in healthy male and female volunteers: a functional magnetic resonance imaging study. Neurosci. Lett. 352, 191-194. doi: 10.1016/j.neulet.2003.08.071

Woodard, J. L., Seidenberg, M., Nielson, K. A., Antuono, P., Guidotti, L., Durgerian, S., et al. (2009). Semantic memory activation in amnestic 
mild cognitive impairment. Brain 132, 2068-2078. doi: 10.1093/brain/ awp 157

Yaffe, K., Petersen, R. C., Lindquist, K., Kramer, J., and Miller, B. (2006). Subtype of mild cognitive impairment and progression to dementia and death. Dement. Geriatr. Cogn. Disord. 22, 312-319. doi: 10.1159/000095427

Yeung, M. K., Han, Y. M., Sze, S. L., and Chan, A. S. (2016). Abnormal frontal theta oscillations underlie the cognitive flexibility deficits in children with highfunctioning autism spectrum disorders. Neuropsychology 30, 281-295. doi: 10. 1037/neu0000231

Zhao, Q., Guo, Q., Li, F., Zhou, Y., Wang, B., and Hong, Z. (2013). The shape trail test: application of a new variant of the trail making test. PLoS One 8:e57333. doi: 10.1371/journal.pone.0057333

Zhao, H., Li, X., Wu, W., Li, Z., Qian, L., Li, S., et al. (2015). Atrophic patterns of the frontal-subcortical circuits in patients with mild cognitive impairment and Alzheimer's disease. PLoS One 10:e130017. doi: 10.1371/journal.pone. 0130017

Conflict of Interest Statement: The authors declare that the research was conducted in the absence of any commercial or financial relationships that could be construed as a potential conflict of interest.

Copyright (c) 2016 Yeung, Sze, Woo, Kwok, Shum, Yu and Chan. This is an openaccess article distributed under the terms of the Creative Commons Attribution License (CC BY). The use, distribution and reproduction in other forums is permitted, provided the original author(s) or licensor are credited and that the original publication in this journal is cited, in accordance with accepted academic practice. No use, distribution or reproduction is permitted which does not comply with these terms. 